\title{
Moduli space of special Lagrangians in almost Kahler spaces
}

\author{
LI MA \\ Department of Mathematical Sciences, Tsinghua University, Beijing 100084, China \\ Manuscript received on August 30, 2000; accepted for publication on November 28, 2000; \\ presented by MANFREDO P. DO CARMO
}

\begin{abstract}
In this work we extend the McLean theorem about the moduli space of special Lagrangian submanifolds in Calabi-Yau (CY) manifolds to almost complex manifolds, which are near to the given CY manifold.
\end{abstract}

Key words: moduli space, special Lagrangians.

\section{INTRODUCTION}

The aim of this work is to generalize the McLean theorem (1998), which is recalled below, to special Lagrangian submanifolds with respect to an admissible family of symplectic manifolds. Therefore, we have to change the complex structure on a Calabi-Yau manifold and introduce some new concepts of variations of complex structures.

Recall that, on the one hand, special Lagrangian submanifolds (to be recalled below) in a Calabi-Yau manifold are important examples in Calibrated Geometry, which was invented by Harvey and Lawson (1982). In fact, these objects in the complex space $C^{n}$ were first studied by them. However, these attracted more attention after the recent work of Strominger, Yau and Zaslow (1996), who proposed an interpretation of mirror symmetry for CY 3-folds M involving fibrations of M by special Lagrangian 3-tori, allowing singular fibres. On the other hand, R. McLean (1998) showed that for a smooth special Lagrangian submanifold $L \subset M$, the moduli space of nearby special Lagrangian submanifolds is smooth and of dimension $b_{1}(L)$, which is the first Betti number of the manifold $L$. An interesting question now is whether this result is also true for a large class of manifolds? Yes, we can do something in this paper.

Our main result is Theorem 1 in section 3 .

E-mail: 1ma@math.tsinghua.edu.cn 


\section{CALIBRATED FORM AND SPECIAL LAGRANGIAN GEOMETRY}

We begin by introducing a Calabi-Yau (in short, CY) structure on an even dimensional manifold in a reverse way in contrast with the standard way.

Let $\Omega$ be a nonvanishing complex valued $n$-form on a real 2 -dimensional manifold $M$. Assume $\Omega$ satisfies the following three conditions:

(1) $\mathrm{d} \Omega=0$,

(2) $\Omega$ is locally decomposable,

(3) $(-1)^{n(n-1) / 2}(i / 2)^{n} \Omega \wedge \bar{\Omega}>0$ everywhere on $M$.

Then, according to Hitchin (1997) (see also Chern (1979)), $\Omega$ determines a complex structure $J$ on $M$ for which $\Omega$ is a holomorphic n-form. We will call such $\Omega$ a CY form. If further, we have a 2 -form $\omega$, which is a symplectic and positive $(1,1)$ form in the complex structure $J$ above, then by defintion, we say that $(\omega, \Omega)$ is a CY structure on $M$. A smooth manifold with a CY structure will be called a CY manifold. According to Hitchin $(1997,1999)$ again, on a CY manifold $M$, the form $\omega$ is a Kahler form whose associated Riemannian metric is Ricci-flat.

Assume that $M$ is a CY manifold with a fixed Riemannian metric $g$ and the structure $(\omega, \Omega)$. Write $\Omega=\alpha+i \beta$, where $\alpha$ and $\beta$ are real and imaginary parts of the form $\Omega$ respectively. By definition, we say that an n-dimensional submanifold $L \subset M$ is a Lagrangian submanifold of $M$ if the restriction of $\omega$ to $L$ is zero. If further, the restriction of $\Omega$ to $L$ is the volume form, then $L$ is called a special Lagrangian submanifold of M. Note that McLean's theorem is within Calabi-Yau geometry.

We now want to change the complex structure of a Calabi-Yau manifold into almost complex structures on M. Hence, we have to introduce some new concepts.

Definition 1. We call $\mathrm{M}$ a pseudo-CY manifold if $\mathrm{M}$ is a symplectic $2 \mathrm{n}$-dimensional manifold endowed with an almost complex structure and a complex valued n-form $\Omega$, whose norm is not greater than one and which has a closed real part, i.e, $d R e \Omega=0$ and satisfies the condition (3).

Definition 2. Assume $M$ is a pseudo-CY manifold. An n-dimensional submanifold $L \subset M$ is called a special Lagrangian submanifold if it is Lagrangian and the restriction to $\mathrm{L}$ of the form $\Omega$ is the volume form on $\mathrm{L}$.

Note that in our definition, special Lagrangian submanifolds are still $R e \Omega$-submanifolds in the almost complex manifold.

We now study a new one-parameter family of complex valued n-forms $\left\{\Omega_{s}\right\}$ such that $\Omega_{0}=\Omega$ is a given $\mathrm{CY}$ form.

DEFINITION 3. Such a family is called admissible if the real part $R e \Omega_{s}$ are closed, the restriction to $L$ of the real 2-form $d /\left.d s\right|_{s=0} \omega_{s}$ is harmonic on $\mathrm{L}$, and the real $\mathrm{n}$-form $d /\left.d s\right|_{s=0} \operatorname{Im}\left(\Omega_{s}\right)$ is a harmonic n-form on L. 
Note that for $s \neq 0$, the $\mathrm{n}$-form $\Omega_{s}$ need not be closed. Therefore, the associated almost complex structures, which will be denoted by $\omega_{s}$, may be non-integrable and the special Lagrangian submanifold is still minimal with respect to the given Riemannian structure on $\left(M, g, \omega_{s}, \Omega_{s}\right)$ where $s \neq 0$. However, we will keep the form $\alpha$ fixed and perturbate the form $\beta$ such that $\Omega_{s}=\alpha+i \beta_{s}$ and in this case, it is clear that we can consider $\alpha:=\operatorname{Re} \Omega$ as the calibrated form and the special lagrangian submanifolds in $\left(M, \omega, \Omega_{s}\right)$ is still minimal. This is a very important example of one-parameter family of special pseudo-CY manifolds.

\section{MODULI SPACE OF SPECIAL LAGRANGIANS}

THEOREM 1. Given a $C Y$ manifold $(M, \omega, \Omega)$ with Riemannian metric g. Suppose $\Omega_{s}$ is an admissible one-parameter family of pseudo-CY forms on $M$ such that $\left.\Omega_{s}\right|_{s=0}=\Omega$. Then there exists a small positive constant $\epsilon_{0}$ such that for any $|s|<\epsilon_{0}$, the moduli space of the special Lagrangian submanifolds near to $L$ is smooth and of dimension $b_{1}(L)$.

REMARK. Before giving a proof of our Theorem, we point out that our result is local in nature, so we need to only assume the existence of the admissible one-parameter family of pseudo-CY forms in a small neighborhood of $L$ in $\mathrm{M}$.

Proof. We denote $N(L)$ the normal bundle of $L$ and $A^{2}(L), A^{n}(L)$ the spaces of differential 2 -forms and $\mathrm{n}$-forms on $\mathrm{L}$ respectively. Let $\exp _{V}$ denote the exponential map which gives a diffeomorphism of L onto its image in the neighborhood of 0 . Since the normal bundle of a special Lagrangian submanifold is isomorphic to the cotangent bundle $T^{*} L$, we have a natural identification of normal vector fields to $L$ with differential 1-forms on L. Furthermore, we identify these normal vector fields with nearby submanifolds.

We now define a map

$$
F: \Gamma(N(L)) \times R \rightarrow A^{2}(L) \times A^{n}(L)
$$

by

$$
\left.F(V, s)=\left(\exp _{V}\right)^{*}\left(-\omega_{s}\right),\left(\exp _{V}\right)^{*} \operatorname{Im}\left(\Omega_{s}\right)\right)
$$

Then the zero set of $F$ is the moduli space of special Lagrangian submanifolds nearby $L$ in the almost complex manifold $\left(M, \omega_{s}, \Omega_{s}\right)$. To use the implicit function theorem (Aubin 1982), we compute

$$
\begin{gathered}
F^{\prime}:=d F(0,0)(V, x)=\left.d_{t}\right|_{t=0, s=0} F(t V, s x) \\
+\left.d_{s}\right|_{t=0, s=0} F(t V, s x),
\end{gathered}
$$

which is

$$
\left(-\left.L_{V} \omega\right|_{L}+x \omega^{\prime}, L_{V} \beta+x \beta^{\prime}\right)
$$

Here

$$
\omega^{\prime}=d /\left.d s \omega\right|_{s=0},
$$


and

$$
\beta^{\prime}=\left.d_{s} \beta\right|_{t=0, s=0}
$$

is the variation of the deformation of the imaginary part of the $(n, 0)$-forms near to $\Omega$. By our assumption, we have that $\omega^{\prime}$ is a harmonic 2-form on $L$ and $\beta^{\prime}$ is a harmonic n-form on $L$. Then as done in McLean's work (1998), we have

$$
F^{\prime}=\left(d v+x \omega^{\prime}, d * v+x \beta^{\prime}\right)
$$

where $v$ is the music dual to the vector field $V$. We may assume that $\beta^{\prime}$ is non-trivial. By Hodge theory we know that $F^{\prime}=0$ if and only if $d v=0, d * v=0, x \omega^{\prime}=0$, and $x \beta^{\prime}=0$ on $L$. As in McLean's work (1998) we can see by using the Poincare's duality that F is surjective on $d A^{1}(L)+H^{2}(L)+d A^{n-1}(L)+H^{n}(L)$. Therefore, we finish the proof of our result.

Here is another example for one parameter family of CY manifolds. Given a CY manifold $(M, \omega, \Omega)$. Let $\Omega_{m}=\exp (\mathrm{im}) \Omega$, where $m$ is a real number. Then $\left(M, \omega, \Omega_{m}\right)$ are CY manifolds and the family $\left(M, \omega, \Omega_{s m}\right)$ is admissible and the theorem above can be applied.

\section{ACKNOWLEDGEMENT}

This work was partially supported by the NSF of China, SRF for Returned Overseas Chinese Scholars, State Education Commission, and a scientific grant of Tsinghua University at Beijing. I would like to thank Profs. Qikeng Lu, K.F. Liu, X.Y. Zhou, and W.P. Zhang, for the invitation for a presentation of this work at the workshop on Mathematical Physics held in July, 1999, in the Morningside Institute of Academia Sinica, Beijing. When we finished our work, we learned that S. Salur (Salur 1999) had a result, but her calibration form may not be closed.

\section{RESUMO}

Neste trabalho, estendemos o teorema de McLean sobre o espaço modular de variedades lagrangianas especiais em variedades de Calabi-Yau (CY) para variedades quasi-complexas que estão próximas a uma dada variedade $\mathrm{CY}$.

Palavras-chave: espaços modulares, Lagrangianos especiais.

\section{REFERENCES}

Aubin TH. 1982. Nonlinear Analysis on Manifolds, Monge-Ampere Equations. Springer-Verlag, Berlin.

CHERn SS. 1979. Complex manifold without potential theory, Springer, Berlin.

Harvey M \& Lawson HB. 1982. Calibrated geometry. Acta Math 148: 47-157.

Hitchin N. 1997. The moduli space of special Lagrangian sub-manifolds. Dedicated to E. DeGiorgi. Ann Scuola Norm Sup Pisa 25: 503-515.

Hitchin N. 1999. Lectures on special Lagrangian submanifolds, ICTP Lecture Note. 
McLeAn R. 1998. Deformations of calibrated submanifolds. Comm Anal Geom 6: 705-747.

SALUR S. 1999. Deformation of special lagrangian submanifolds, Math.DG/9906048.

Strominger A, Yau ST, Zaslow E. 1996. Mirror symmetry is T-duality. Nucl Phys B 479: 243-259. 